\title{
A FAMILY OF RANDOM MAPS WHICH POSSES INFINITE ABSOLUTELY CONTINUOUS INVARIANT MEASURES
}

\author{
MD SHAFIQUL ISLAM \\ School of Mathematical and Computational Sciences \\ University of Prince Edward Island \\ 550 University Ave, Charlottetown, PE, C1A 4P3, CANADA
}

\begin{abstract}
We consider a family of random maps where each of the component maps is from a family of piecewise, linear and Markov maps on a class of infinite partitions of the state space. We investigate the existence of infinite absolutely continuous invariant measures of the random maps. In a more general setting, our study establishes a positive answer to the question in discrete time dynamical system: can two chaotic systems give rise to order, namely can they be combined into another dynamical system which does not behave chaotically? This question is analogous to Parrondo's paradox [5] which states that two losing gambling games when combined one after the other (either deterministically or randomly) can result in a winning game: that is, a losing game followed by a losing game = a winning game.
\end{abstract}

AMS Subject Classification: $37 \mathrm{~A} 05,37 \mathrm{H} 10$

Key Words: family of random maps, piecewise, linear and Markov maps, Parrondo's paradox

Received: $\quad$ March 16, 2018;

Published: September 6, 2018
Accepted: August 22, 2018;

doi: $\quad 10.12732 /$ dsa.v27i4.3

\section{INTRODUCTION}

The existence of infinite absolutely continuous invariant measures (acim) for random maps is one of the important and challenging problems in ergodic theory and dynamical systems. For deterministic maps, the existence of finite absolutely continuous invariant measures is well studied by many authors (see, for example [9] and the references therein). Farey map is an infinite measure preserving transformation which is defined on an infinite partition $\alpha$ (see [3, 4]). Infinite ergodic theory for 
deterministic maps and properties of infinite measure preserving transformations are studied by J. Aaronson in [1]. The main objective of this paper is to study infinite acims for random maps. We consider a family of random maps of piecewise linear and Markov maps on a class of infinite partitions of the state space. The existence of infinite acim for the random maps is established via the study of the corresponding Frobenius-Perron operators of the random maps.

Let $(X, \mathcal{B}, \lambda)$ be a measure space, where $\mathcal{B}$ is a $\sigma$-algebra on $X$ and $\lambda$ is the Lebesgue measure on $\mathcal{B}$. Let $\Omega=\{1,2,3, \ldots, K\}^{\{0,1,2, \ldots\}}=\left\{\omega=\left\{\omega_{i}\right\}_{i=0}^{\infty}: \omega_{i} \in\right.$ $\{1,2,3, \ldots, K\}\}$ be the set of set of all one sided infinite sequences. Let $\tau_{k}: X \rightarrow$ $X, k=1,2, \ldots, K$ be nonsingular piecewise one-to-one transformations and $p_{1}, p_{2}, \ldots$, $p_{K}$ be constant probabilities such that $\sum_{i=1}^{K} p_{i}=1$. The topology on $\Omega$ is the product of the discrete topology on $\{1,2,3, \ldots, n\}$ and the Borel probability measure $\mu_{p}$ on $\Omega$ is defined as $\mu_{p}\left(\left\{\omega: \omega_{0}=i_{0}, \omega_{1}=i_{1}, \ldots, \omega_{n}=i_{n}\right\}\right)=p_{i_{0}} p_{i_{1}} \ldots p_{i_{n}}$. Let $\sigma: \Omega \rightarrow \Omega$ be the left shift. Consider the skew product $S: \Omega \times X \rightarrow \Omega \times X$ defined by

$$
S(\omega, x)=\left(\sigma(\omega), \tau_{\omega_{0}}(x)\right), \omega \in \Omega, x \in X
$$

Now,

$$
S^{2}(\omega, x)=\left(\sigma^{2}(\omega), \tau_{\omega_{1}} \circ \tau_{\omega_{0}}(x)\right)
$$

and for any integer $N \geq 1$,

$$
S^{N}(\omega, x)=\left(\sigma^{N}(\omega), \tau_{\omega_{N-1}} \circ \tau_{\omega_{N-2}} \circ \ldots \circ \tau_{\omega_{1}} \circ \tau_{\omega_{0}}(x)\right)
$$

A random map

$$
T=\left\{\tau_{1}, \tau_{2}, \ldots, \tau_{K} ; p_{1}, p_{2}, \ldots, p_{K}\right\}
$$

with constant probabilities $p_{1}, p_{2}, \ldots, p_{K}$ is defined as follows: for any $x \in X, T(x)=$ $\tau_{k}(x)$ with probability $p_{k}$ and for any non-negative integer $N, T^{N}(x)=\tau_{k_{N}} \circ \tau_{k_{N-1}} \circ$ $\ldots \circ \tau_{k_{1}}(x)$ with probability $\Pi_{j=1}^{N} p_{k_{j}} . T^{N}(x)$ can be viewed as the second component of the $S^{N}$ of the skew product $S$. A measure $\mu$ is invariant under the random map $T$ if

$$
\mu(E)=\sum_{k=1}^{K} p_{k} \mu\left(\tau_{k}^{-1}(E)\right),
$$

for any measurable set $E \in \mathcal{B}$. It can be easily shown that a measure $\mu$ is $T$ - invariant if and only if the measure $\mu_{p} \times \mu$ is $S$-invariant. The Perron-Frobenius operator $P_{T}$ for the random map $T$ is given by

$$
P_{T} f=\sum_{k=1}^{K} p_{k} P_{\tau_{k}} f,
$$

where $P_{\tau_{k}}$ is the Frobenius-Perron operator of the transformation $\tau_{k}$. For random maps with constant probabilities where the component maps are Lasota-Yorke maps 
[6], Pelikan [7] proved the following sufficient condition for the existence of an acim:

$$
\sum_{k} \frac{p_{k}}{\left|\tau_{k}^{\prime}(x)\right|} \leq \alpha<1
$$

for all $x \in I$.

\section{A FAMILY OF RANDOM MAPS WHICH ADMITS AN INFINITE ABSOLUTELY CONTINUOUS INVARIANT MEASURE}

Let

$$
\alpha:=\left\{I_{n}^{(1)}: n \in \mathbb{N}\right\}
$$

be a countable infinite partition of $\left[0, \frac{1}{2}\right)$ where each $I_{n}^{(1)}, n=1,2, \cdots$ is a non-empty interval of of the form $[a, b)$. We assume that the elements of $\alpha$ are ordered from left to right and the interval $I_{n}^{(1)}$ approaches to the point $\frac{1}{2}$ as $n \rightarrow \infty$. Similarly, let

$$
\beta:=\left\{I_{n}^{(2)}: n \in \mathbb{N}\right\}
$$

be a countable infinite partition of $\left[\frac{1}{2}, 1\right]$, where each $I_{n}^{(2)}, n=1,2, \cdots$ is a non-empty interval of the form $[c, d)$. We assume that the elements of $\beta$ are ordered from left to right and the interval $I_{n}^{(2)}$ approaches to the point 1 as $n \rightarrow \infty$. Consider the partition $\mathcal{P}=\{\alpha, \beta\}$ of $[0,1]$. We construct families of piecewise linear Markov maps $\tau_{1, \alpha, \beta}$ and $\tau_{2, \alpha, \beta}$ (w. r. t. $\mathcal{P}$ ) on $[0,1]$ into itself as follows: let

$$
\begin{gathered}
a_{n}=\lambda\left(I_{n}^{(1)}\right), \quad s_{n}=\sum_{k=1}^{n} a_{k}, n=1,2, \cdots . \\
b_{n}=\lambda\left(I_{n}^{(2)}\right), \quad t_{n}=\frac{1}{2}+\sum_{k=1}^{n} b_{k}, n=1,2, \cdots .
\end{gathered}
$$

Fix integers $i^{*} \geq 1, k^{*} \geq 1, m^{*} \geq 1, n^{*} \geq 1$. Define $\tau_{1, \alpha, \beta}, \tau_{2, \alpha, \beta}:[0,1] \rightarrow[0,1]$ by 


$$
\begin{aligned}
& \tau_{1, \alpha, \beta}(x)= \begin{cases}\frac{t_{i^{*}+1}-t_{i^{*}}}{s_{1}} x+t_{i^{*}}, & x \in A_{1} ; \\
\frac{t_{i^{*}+k}-t_{i^{*}+k-1}}{s_{k}-s_{k-1}}\left(x-s_{k-1}\right)+t_{i^{*}+(k-1)}, & x \in A_{k}, k \geq 2 ; \\
1, & x=\frac{1}{2} ; \\
\frac{\frac{1}{2}\left(x-\frac{1}{2}\right)}{\frac{1}{2}-t_{1}}+1, & x \in B_{1} ; \\
\frac{\frac{1}{2}-t_{k^{*}}}{t_{1}-t_{2}}\left(x-t_{1}\right)+\frac{1}{2}, & x \in B_{2} ; \\
\frac{t_{k^{*}+k-1}-t_{k^{*}+(k-2)}}{t_{1+k}-t_{k}}\left(x-t_{k}\right)+t_{k^{*}+k-2}, & x \in B_{1+k}, k \geq 2 ; \\
1, & x=1 .\end{cases} \\
& \tau_{2, \alpha, \beta}(x)= \begin{cases}\frac{-\frac{1}{2}}{s_{1}} x+\frac{1}{2}, & x \in A_{1} ; \\
\frac{s_{m^{*}}}{s_{2}-s_{1}}\left(x-s_{1}\right), & x \in A_{2} ; \\
\frac{s_{m^{*}+k-1}-s_{m^{*}+k-2}}{s_{1+k}-s_{k}}\left(x-s_{k}\right)+s_{m^{*}+k-2}, & x \in A_{1+k}, k \geq 2 ; \\
\frac{1}{2}, & x=\frac{1}{2} ; \\
\frac{s_{n^{*}+1}-s_{n^{*}}}{t_{1}-\frac{1}{2}}\left(x-\frac{1}{2}\right)+s_{n^{*}}, & x \in B_{1} ; \\
\frac{s_{n^{*}+k+1}-s_{n^{*}+k}}{t_{k+1}-t_{k}}\left(x-t_{k}\right)+s_{n^{*}+k}, & x \in B_{k+1}, k \geq 1 ; \\
\frac{1}{2}, & x=1 .\end{cases}
\end{aligned}
$$

For any partition $\mathcal{P}=\{\alpha, \beta\}$ defined above and any probability $p$, consider the family $\left\{T_{\alpha, \beta}\right\}$ of random maps

$$
T_{\alpha, \beta}=\left\{\tau_{1, \alpha, \beta}, \tau_{2, \alpha, \beta} ; p, 1-p\right\},
$$

where $\tau_{1, \alpha, \beta}$ and $\tau_{2, \alpha, \beta}$ are defined in (2.5). It is easy to see that each of maps $\left\{\tau_{1, \alpha, \beta}, \tau_{2, \alpha, \beta}\right\}$ admits a finite acim. The map $\tau_{1, \alpha, \beta}$ preserves Lebesgue measure on $[1 / 2,1]$ and $\tau_{2, \alpha, \beta}$ preserves Lebesgue measure on $[0,1 / 2]$. The composition $\tau_{1, \alpha, \beta} \circ$ $\tau_{2, \alpha, \beta}$ has an attracting fixed point 1 as a global attractor and the other composition $\tau_{2, \alpha, \beta} \circ \tau_{1, \alpha, \beta}$ has an attracting fixed point $1 / 2$ as a global attractor. Thus, the periodic switching of these maps leads to completely regular dynamics. We can describe the periodic switching of $\tau_{1, \alpha, \beta}$ and $\tau_{2, \alpha, \beta}$ as "trap-rescue" dynamics : iteration of $\tau_{1, \alpha, \beta}$ traps trajectory in $[1 / 2,1]$ and iteration of $\tau_{2, \alpha, \beta}$ traps trajectory in $[0,1 / 2]$. Then, periodic application of the these maps rescues the trajectory consecutively from each of these intervals creating different, nearly ordered dynamics.

Example 2.1. Let

$$
\alpha=\left\{\left[\frac{1}{2}-\frac{1}{2^{k-1}}, \frac{1}{2}-\frac{1}{2^{k}}\right]\right\}, k=2,3,4, \ldots
$$

and

$$
\beta=\left\{\left[1-\frac{1}{2^{k-1}}, 1-\frac{1}{2^{k}}\right]\right\}, k=2,3,4, \ldots
$$




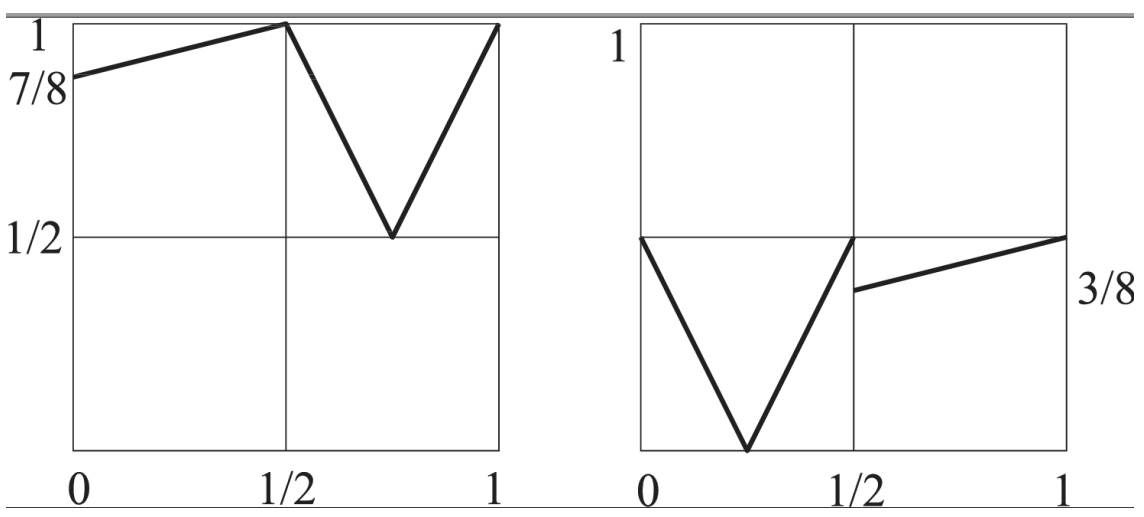

Figure 1: The graphs of $\tau_{1}$ and $\tau_{2}$.

Then:

$$
\begin{gathered}
a_{k-1}=\frac{1}{2^{k}}, k=2,3, \cdots, \text { i.e., } a_{1}=\frac{1}{4}, a_{2}=\frac{1}{8}, a_{3}=\frac{1}{16}, \cdots \\
b_{k-1}=\frac{1}{2^{k}}, k=2,3, \cdots, \text { i.e., } b_{1}=\frac{1}{4}, b_{2}=\frac{1}{8}, b_{3}=\frac{1}{16}, \cdots \\
s_{1}=\frac{1}{4}, s_{2}=\frac{3}{8}, s_{3}=\frac{7}{16}, \cdots \\
t_{1}=\frac{3}{4}, t_{2}=\frac{7}{8}, t_{3}=\frac{15}{16}, \cdots .
\end{gathered}
$$

Fix $i^{*}=2, k^{*}=1, m^{*}=1, n^{*}=2$. Then the random map in (2.6) reduces to the random map $T=\left\{\tau_{1}, \tau_{2} ; p_{1}, p_{2}\right\}$, where $\tau_{1}, \tau_{2}:[0,1] \rightarrow[0,1]$ (see Fig. 1 and [2]) are defined by

$$
\begin{gathered}
\tau_{1}(x)= \begin{cases}\frac{1}{4} x+\frac{7}{8}, & \text { for } 0 \leq x<\frac{1}{2} \\
1, & \text { for } x=\frac{1}{2} \\
2-2 x, & \text { for } \frac{1}{2} \leq x<\frac{3}{4} \\
2 x-1, & \text { for } \frac{3}{4} \leq x<1 \\
1, & \text { for } x=1\end{cases} \\
\tau_{2}(x)= \begin{cases}\frac{1}{2}-2 x, & \text { for } 0 \leq x<\frac{1}{4} \\
2 x-\frac{1}{2}, & \text { for } \frac{1}{4} \leq x<\frac{1}{2} \\
\frac{1}{2}, & \text { for } x=\frac{1}{2} \\
\frac{1}{4} x+\frac{1}{4}, & \text { for } \frac{1}{2}<x<1 \\
\frac{1}{2}, & \text { for } x=1\end{cases}
\end{gathered}
$$


Theorem 2.2. Let $\mathcal{P}=\{\alpha, \beta\}$ be a partition of $[0,1]$, where $\alpha$ and $\beta$ are defined in (2.1) and (2.2) respectively. Let $a_{n}, b_{n}$ are sequences defined in (2.3) (2.4) such that $a_{n}=b_{n}$ for each $n=1,2, \ldots$ Let $\tau_{1, \alpha, \beta}, \tau_{2, \alpha, \beta}$ are maps defined in (2.5) satisfying $i^{*}=n^{*}$ and $k^{*}=m^{*}$. Then for any probability $p$, the random map $T_{\alpha, \beta}=\left\{\tau_{1, \alpha, \beta}, \tau_{2, \alpha, \beta} ; p, 1-p\right\}$ in (2.6) admits an ergodic infinite absolutely continuous invariant measure and it has no finite absolutely continuous invariant measure.

Proof. The Perron-Frobenius operators of $\tau_{1, \alpha, \beta}$ and $\tau_{2, \alpha, \beta}$ can be represented as matrices [9, Chapter 9]:

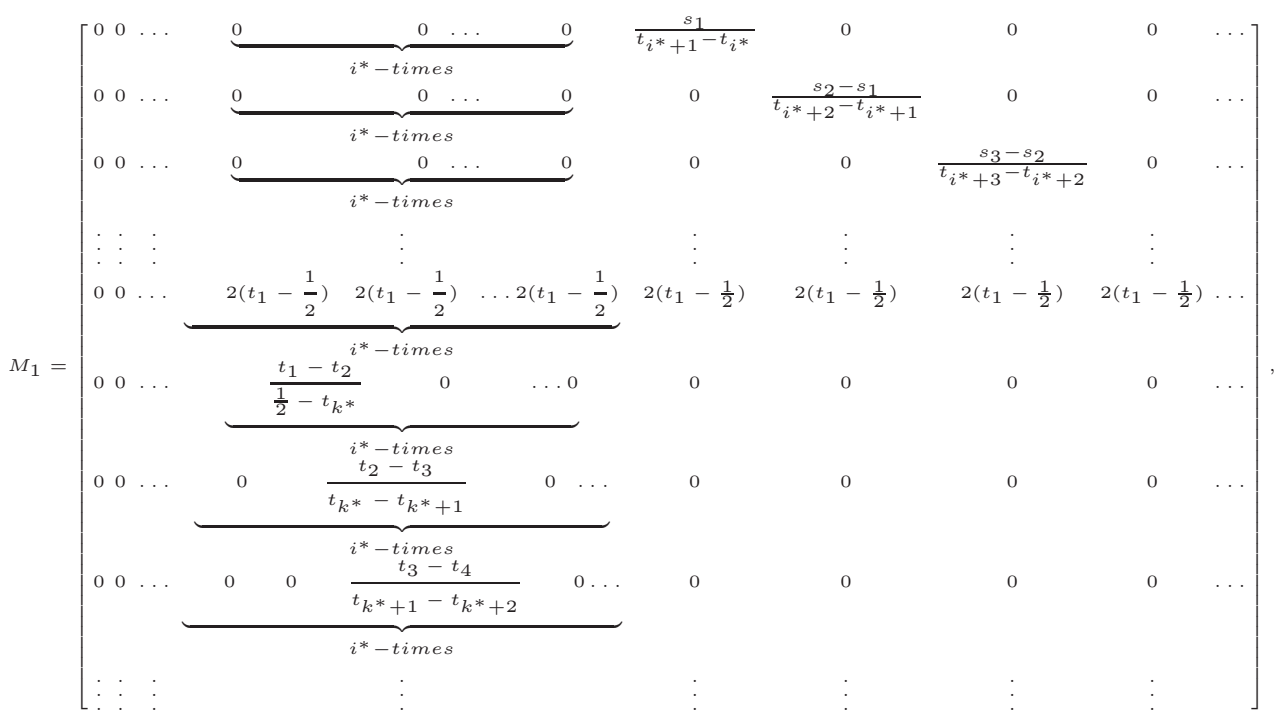

and

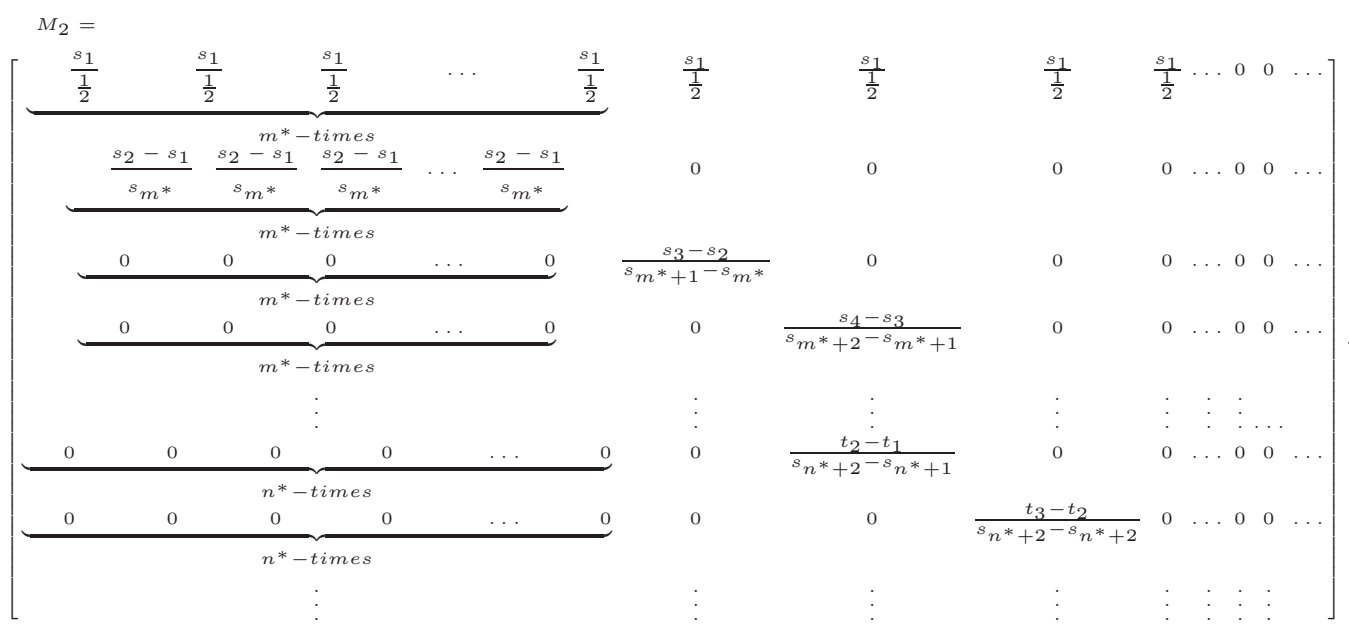

Without the loss of generality and for the convenience of the calculation, we fix 
$i^{*}=2, k^{*}=1, m^{*}=1$ and $n^{*}=2$. Then, the above matices reduce to

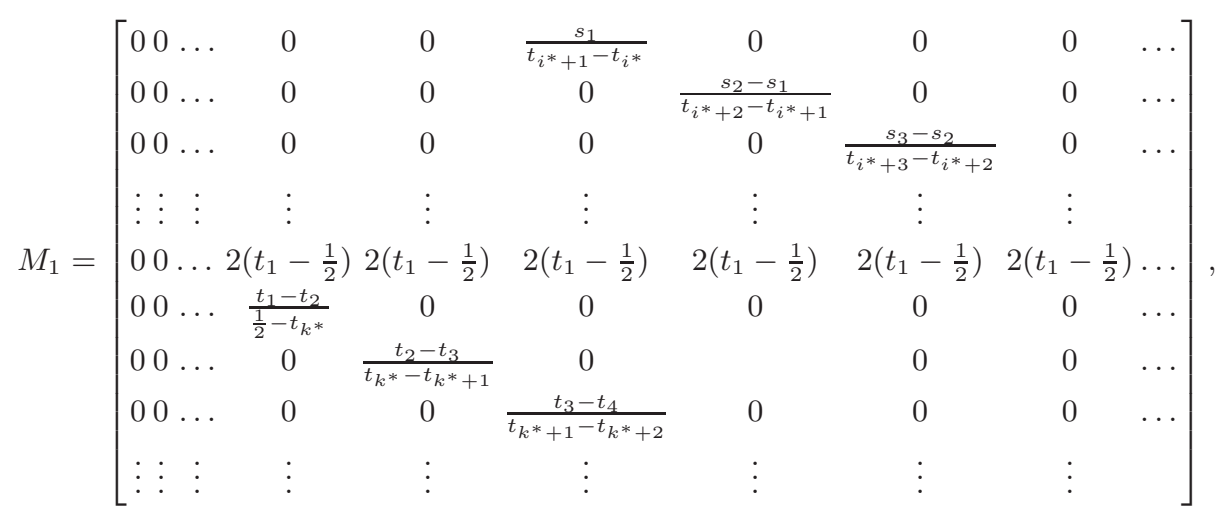

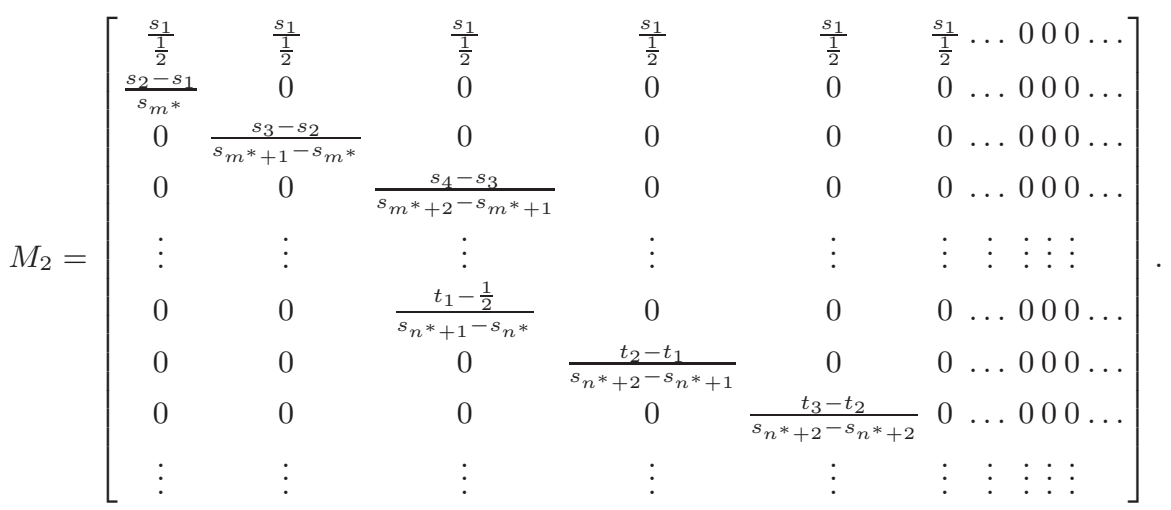

Let the invariant density of the random map $T_{\alpha, \beta}=\left\{\tau_{1, \alpha, \beta}, \tau_{2, \alpha, \beta} ; p, 1-p\right\}$ be piecewise constant on the elements of the partition $\mathcal{P}$ and represented by an infinite vector $f=\left[x_{i *}, x_{i^{*}+1}, x_{i^{*}+2}, \ldots, y_{n *}, y_{n *+1}, y_{n^{*}+2}, \ldots,\right]$. Then,

$$
f=(p f) \cdot M_{1}+((1-p) f) \cdot M_{2}
$$


From equation (2.9), we get:

$$
\begin{aligned}
& x_{i^{*}+1}=\frac{s_{m^{*}}}{s_{2}-s_{1}} \cdot \frac{1-2 s_{1}+2 s_{1} p}{1-p} \cdot x_{i^{*}}, \\
& x_{i^{*}+2}=\frac{s_{m^{*}+1}-s_{m^{*}}}{s_{3}-s_{2}} \cdot\left[\frac{s_{m^{*}}}{s_{2}-s_{1}} \cdot \frac{1-2 s_{1}+2 s_{1} p}{(1-p)^{2}}-2 s_{1}\right] \cdot x_{i^{*}}, \\
& x_{i^{*}+3}=\frac{s_{m^{*}+2}-s_{m^{*}+1}}{s_{4}-s_{3}} \cdot\left[\frac{1}{1-p} \cdot x_{i^{*}+2}-\frac{t_{1}-\frac{1}{2}}{s_{n^{*}+1}-s_{n^{*}}} \cdot y_{n^{*}}-2 s_{1} \cdot x_{i^{*}}\right], \\
& x_{i}=\frac{s_{m^{*}+i-3}-s_{m^{*}+i-4}}{s_{i-1}-s_{i-2}} \cdot\left[\frac{1}{1-p} \cdot x_{i-1}-\frac{t_{i-3}-t_{i-4}}{s_{n^{*}+i-4}-s_{n^{*}+i-5}} \cdot y_{i-3}-2 s_{1} \cdot x_{i^{*}}\right] \text {, } \\
& i=i^{*}+4, i^{*}+5, \ldots \\
& y_{n^{*}+1}=\frac{\frac{1}{2}-t_{k^{*}}}{t_{1}-t_{2}}\left[\frac{1}{p}-2\left(t_{1}-\frac{1}{2}\right)\right] \cdot y_{n^{*}}, \\
& y_{n^{*}+2}=\frac{t_{k^{*}}-t_{k^{*}+1}}{t_{2}-t_{3}} \cdot\left[\frac{\frac{1}{2}-t_{k^{*}}}{t_{1}-t_{2}} \cdot\left(\frac{1}{p^{2}}-\frac{2\left(t_{1}-\frac{1}{2}\right)}{p}-2\left(t_{1}-\frac{1}{2}\right)\right)\right] y_{n^{*}}, \\
& y_{n^{*}+3}=\frac{t_{k^{*}+1}-t_{k^{*}+2}}{t_{3}-t_{4}} \cdot\left[\frac{1}{p} \cdot y_{n^{*}+2}-\frac{s_{2}-s_{1}}{t_{i^{*}+1}-t_{i^{*}}} x_{i^{*}}-2\left(t_{1}-\frac{1}{2}\right) y_{n^{*}}\right] \text {, } \\
& y_{i}=\frac{t_{k^{*}+i-4}-t_{k^{*}+i-3}}{t_{i-2}-t_{i-1}} \cdot\left[\frac{1}{p} \cdot y_{i-1}-\frac{s_{i-4}-s_{i-5}}{t_{i^{*}+i-3}-t_{i^{*}+i-4}} x_{i-3}-2\left(t_{1}-\frac{1}{2}\right) y_{n^{*}}\right] \text {, } \\
& i=n^{*}+4, n^{*}+5, \ldots
\end{aligned}
$$

Consider the following change of variables

$$
u_{i}=x_{i-1}, v_{i}=y_{i-1}, w_{i}=u_{i-1}, p_{i}=v_{i-1}, a_{i}=w_{i-1}, b_{i}=p_{i-1} .
$$

Then (2.10) reduces to

$$
\begin{aligned}
x_{j}= & \frac{s_{m^{*}+j-3}-s_{m^{*}+j-4}}{s_{j}-s_{j-1}} \cdot\left[\frac{1}{1-p} \cdot x_{j-1}-\frac{t_{j-4}-t_{j-5}}{s_{n^{*}+j-4}-s_{n^{*}+j-5}} \cdot p_{j-1}-2 s_{1} \cdot x_{i^{*}}\right], \\
& \quad j=i^{*}+4, i^{*}+5, \ldots, \\
y_{j}= & \frac{t_{k^{*}+j-4}-t_{k^{*}+j-3}}{t_{j-1}-t_{j}} \cdot\left[\frac{1}{p} \cdot y_{j-1}-\frac{s_{j-4}-s_{j-5}}{t_{i^{*}+j-4}-t_{i^{*}+j-5}} w_{j-1}-2\left(t_{1}-\frac{1}{2}\right) y_{n^{*}}\right], \\
& \quad j=n^{*}+4, n^{*}+5, \ldots, \\
u_{j}= & x_{j-1} \\
v_{j}= & y_{j-1}, \\
w_{j}= & u_{j-1}, \\
p_{j}= & v_{j-1}
\end{aligned}
$$

Let $X_{i}=\left(x_{j}, y_{j}, u_{j}, v_{i}, w_{j}, p_{j}\right)$, 


$$
A=
$$

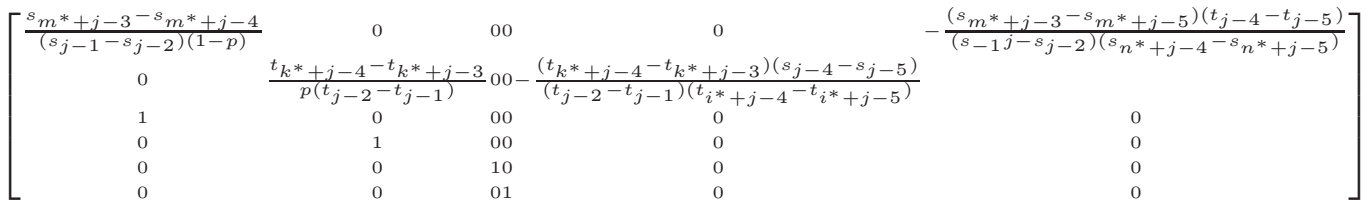

and $b=\left(-2 s_{1}\left(\frac{s_{m^{*}+j-3}-s_{m^{*}+j-4}}{s_{j-1}-s_{j-2}}\right) \cdot x_{i}^{*},-2\left(t_{1}-\frac{1}{2}\right) \cdot \frac{t_{k^{*}+j-4}-t_{k^{*}+j-3}}{t_{j-1}-t_{j}} \cdot y_{n}^{*}, 0,0,0,0\right)$. If $i^{*}=n^{*}$ and $k^{*}=m^{*}$ then the matrix $A$ and the vector $b$ is constant for each $j$. In particular, $i^{*}=n^{*}=2$ and $k^{*}=m^{*}=1$, then

$$
A=\left[\begin{array}{cccccc}
\frac{2}{(1-p)} & 0 & 0 & 0 & 0 & -8 \\
0 & \frac{2}{p} & 0 & 0 & -8 & 0 \\
1 & 0 & 0 & 0 & 0 & 0 \\
0 & 1 & 0 & 0 & 0 & 0 \\
0 & 0 & 1 & 0 & 0 & 0 \\
0 & 0 & 0 & 1 & 0 & 0
\end{array}\right]
$$

and $b=\left(-x_{2},-y_{2}, 0,0,0,0\right)$. Then, the system (2.11) reduces to

$$
X_{j}=A X_{j-1}+b
$$

The system (2.12) has the following form of solution [10]:

$$
X_{j}=A^{j} X_{0}+\sum_{k=0}^{j} A^{j-k} b .
$$

Note that one of the eigenvalues of the matrix $A$ in the system (2.12) is exactly 2 . Hence, the maximal eigenvalue $\gamma$ is larger than 2. Thus, $x_{j}$ and $y_{j}$ in $(2.10)$ are of the form constant $\cdot \gamma^{i}$ and the densities blow up in the $\epsilon$-neighborhood of $\frac{1}{2}$ and 1 . Moreover, $\sum_{i=2}^{\infty} \sum_{k=1,2} x_{i}^{(k)} \lambda\left(I_{i}^{(k)}\right)=\infty$. In particular, and

$$
\begin{aligned}
\sum_{i=2}^{\infty} \sum_{k=1,2} x_{i}^{(k)} \lambda\left(I_{i}^{(k)}\right)= & {\left[x_{2} \cdot \frac{1}{2^{2}}+x_{3} \cdot \frac{1}{2^{3}}+x_{4} \cdot \frac{1}{2^{4}}+x_{5} \cdot \frac{1}{2^{5}} \ldots\right] } \\
& +\left[y_{2} \cdot \frac{1}{2^{2}}+y_{3} \cdot \frac{1}{2^{3}}+y_{4} \cdot \frac{1}{2^{4}}+y_{5} \cdot \frac{1}{2^{5}} \ldots\right] \\
= & \infty .
\end{aligned}
$$

This means that the random map has an infinite acim $m=f \cdot \lambda$.

Now, we prove that the measure $m=f \cdot \lambda$ is ergodic. It is enough to show that the induced Frobenius-Perron matrix is irreducible [8]. The transition graph of the induced Frobenius-Perron matrix of the random map $\mathrm{T}$ in Example 2 is shown in Fig 2. It is easy to see that the graph is strongly connected, i.e., every state communicates with every other. Thus, the matrix $M$ is irreducible and the measure $m$ is ergodic. 


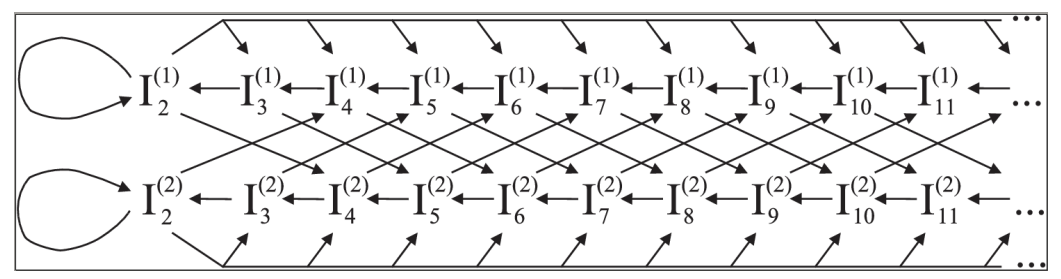

Figure 2: The transition graph of the induced Frobenius-Perron operator of the random map $T$.

Since $m$ is supported on all of [0,1], there is no other acim. In particular, this random map does not admit a finite acim.

\section{EXAMPLE}

Example 3.1. Let

$$
\alpha=\left\{\left[\frac{1}{2}-\frac{1}{2^{k-1}}, \frac{1}{2}-\frac{1}{2^{k}}\right]\right\}, k=2,3,4, \ldots
$$

and

$$
\beta=\left\{\left[1-\frac{1}{2^{k-1}}, 1-\frac{1}{2^{k}}\right]\right\}, k=2,3,4, \ldots
$$

Then,

$$
\begin{gathered}
a_{k-1}=\frac{1}{2^{k}}, k=2,3, \cdots, \text { i.e., } a_{1}=\frac{1}{4}, a_{2}=\frac{1}{8}, a_{3}=\frac{1}{16}, \cdots, \\
b_{k-1}=\frac{1}{2^{k}}, k=2,3, \cdots, \text { i.e., } b_{1}=\frac{1}{4}, b_{2}=\frac{1}{8}, b_{3}=\frac{1}{16}, \cdots . \\
s_{1}=\frac{1}{4}, s_{2}=\frac{3}{8}, s_{3}=\frac{7}{16}, \cdots, \\
t_{1}=\frac{3}{4}, t_{2}=\frac{7}{8}, t_{3}=\frac{15}{16}, \cdots .
\end{gathered}
$$

Fix $i^{*}=3, k^{*}=1, m^{*}=1, n^{*}=3$. Then the random map in (2.6) reduces to the random map $T=\left\{\tau_{1}, \tau_{2} ; \frac{1}{2}, \frac{1}{2}\right\}$, where $\tau_{1}, \tau_{2}:[0,1] \rightarrow[0,1]$ (see Fig. 1) are defined by

$$
\tau_{1}(x)= \begin{cases}\frac{1}{8} x+\frac{15}{16}, & \text { for } 0 \leq x<\frac{1}{2} \\ 1, & \text { for } x=\frac{1}{2} \\ 2-2 x, & \text { for } \frac{1}{2} \leq x<\frac{3}{4} \\ 2 x-1, & \text { for } \frac{3}{4} \leq x<1 \\ 1, & \text { for } x=1\end{cases}
$$




$$
\tau_{2}(x)= \begin{cases}\frac{1}{2}-2 x, & \text { for } 0 \leq x<\frac{1}{4} \\ 2 x-\frac{1}{2}, & \text { for } \frac{1}{4} \leq x<\frac{1}{2} \\ \frac{1}{2}, & \text { for } x=\frac{1}{2} \\ \frac{1}{8} x+\frac{3}{8}, & \text { for } \frac{1}{2} \leq x<1 \\ \frac{1}{2}, & \text { for } x=1\end{cases}
$$

The Perron-Frobenius operators of $\tau_{1}$ and $\tau_{2}$ can be represented as matrices [9, Chapter 9]:

$$
M_{1}=\left[\begin{array}{ccccccccccc}
0 & 0 & \ldots & 0 & 0 & 0 & 8 & 0 & 0 & 0 & \ldots \\
0 & 0 & \ldots & 0 & 0 & 0 & 0 & 8 & 0 & 0 & \ldots \\
0 & 0 & \ldots & 0 & 0 & 0 & 0 & 0 & 8 & 0 & \ldots \\
\vdots & \vdots & \vdots & \vdots & \vdots & \vdots & \vdots & \vdots & \vdots & \vdots & \vdots \\
0 & 0 & \ldots & \frac{1}{2} & \frac{1}{2} & \frac{1}{2} & \frac{1}{2} & \frac{1}{2} & \frac{1}{2} & \frac{1}{2} & \ldots \\
0 & 0 & \ldots & \frac{1}{2} & 0 & 0 & 0 & 0 & 0 & 0 & \ldots \\
0 & 0 & \ldots & 0 & \frac{1}{2} & 0 & 0 & 0 & 0 & 0 & \ldots \\
0 & 0 & \ldots & 0 & 0 & \frac{1}{2} & 0 & 0 & 0 & 0 & \ldots \\
\vdots & \vdots & \vdots & \vdots & \vdots & \vdots & \vdots & \vdots & \vdots & \vdots & \vdots
\end{array}\right]
$$

An invariant density $f$ of the random map $T=\left\{\tau_{1}, \tau_{2} ; \frac{1}{2}, \frac{1}{2}\right\}$ satisfies the following Perron-Frobenius equation:

$$
f=\frac{1}{2}\left\{f M_{1}+f M_{2}\right\}
$$


Let

$$
M=M_{1}+M_{2}=\left[\begin{array}{cccccccccccc}
\frac{1}{2} & \frac{1}{2} & \frac{1}{2} & \frac{1}{2} & \frac{1}{2} & \ldots & 0 & 0 & 0 & 8 & 0 & \ldots \\
\frac{1}{2} & 0 & 0 & 0 & 0 & \ldots & 0 & 0 & 0 & 0 & 8 & \ldots \\
0 & \frac{1}{2} & 0 & 0 & 0 & \ldots & 0 & 0 & 0 & 0 & 0 & \ldots \\
\vdots & \vdots & \vdots & \vdots & \vdots & \vdots & \vdots & \vdots & \vdots & & & \\
0 & 0 & 0 & 8 & 0 & \ldots & \frac{1}{2} & \frac{1}{2} & \frac{1}{2} & \frac{1}{2} & \ldots & \\
0 & 0 & 0 & 0 & 8 & \ldots & \frac{1}{2} & 0 & 0 & 0 & 0 & \ldots \\
0 & 0 & 0 & 0 & 0 & \ldots & 0 & \frac{1}{2} & 0 & 0 & 0 & \ldots \\
\vdots & \vdots & \vdots & \vdots & \vdots & \vdots & \vdots & \vdots & \vdots & \vdots & \vdots & \vdots
\end{array}\right]
$$

Then (3.3) reduces to

$$
2 f=f M \text {. }
$$

If the value of the invariant density $f$ on $I_{k}^{(i)}$ is constant $x_{k}^{(i)}$ and we assume that $x_{k}^{(1)}=x_{k}^{(2)}=x_{k}$, for all $k=2,3, \ldots$, then the density $f$ can be represented as an infinite vector $\left(x_{2}, x_{3}, \ldots, x_{2}, x_{3}, \ldots\right)$ and equation (3.4) can be written as:

$$
2\left(x_{2}, x_{3}, \ldots, x_{2}, x_{3}, \ldots\right)=\left(x_{2}, x_{3}, \ldots, x_{2}, x_{3}, \ldots\right) M .
$$

From equation (3.5), we get:

$$
\begin{aligned}
x_{3} & =3 x_{2} ; \\
x_{4} & =11 x_{2} ; \\
x_{5} & =43 x_{2} ; \\
x_{i} & =4 x_{i-1}-16 x_{i-4}-x_{2}, \quad i=6,7, \ldots
\end{aligned}
$$

From the above system we get

$$
x_{3}=3 \cdot x_{2}, x_{4}=11 \cdot x_{2}, x_{5}=43 \cdot x_{2}, x_{6}=155 \cdot x_{2}, x_{7}=571 \cdot x_{2}, \ldots
$$

Now, we find a general solution of the system (3.6) of discrete difference equations. Consider the homogeneous equation $x_{i}=4 x_{i-1}-16 x_{j-4}$. Let $x_{i}=\beta^{i} x_{2}$. Let $\beta_{1,2,3,4}$ be the roots of $\beta^{4}-4 \beta^{3}+16=0$. The roots are $\beta_{1}=2, \beta_{2}=3.67857$, $\beta_{3}=-0.839287+1.21258 i, \beta_{4}=\overline{\beta_{3}}$, where $\overline{\beta_{3}}$ represents complex conjugation of $\beta_{3}$. The general solution of the difference equation will be

$$
x_{i}=\left(c_{1} \beta_{1}^{i}+c_{2} \beta_{2}^{i}+c_{3} \beta_{3}^{i}+c_{4} \beta_{4}^{i}\right) x_{2} .
$$

Calculating $c_{1,2,3,4}$ leads to the values $c_{1}=0, c_{2}=0.0627625, c_{3}=0.00708027+$ $0.0585811 \sqrt{-1}, c_{4}=\overline{c_{3}}$. Since $c_{1}=0$ we can omit the first term in the general form of $x_{i}$. We now show that each $x_{i}$ is a real number by using that $c_{4} \beta_{4}^{i}=\overline{c_{3} \beta_{3}^{i}}$ and that $c_{3} \beta_{3}^{i}+\overline{c_{3} \beta_{3}^{i}}=2 \operatorname{Re}\left(c_{3} \beta_{3}^{i}\right)$ in order to write

$$
x_{i}=c_{2} \beta_{2}^{i}+2 \operatorname{Re}\left(c_{3} \beta_{3}^{i}\right) .
$$


To show that the series $\sum_{j=2}^{\infty} \sum_{k=1,2} x_{i}^{(k)} \lambda\left(I_{i}^{(k)}\right)$ we show that $\sum_{i=1}^{\infty} \frac{x_{i}}{2^{i+1}}$ diverges. We use the limit comparison test with the divergent geometric series $\sum \frac{\beta_{2}^{i}}{2^{i+1}}$. First note the inequality

$$
\left(c_{2} \beta_{2}^{i}-2\left|c_{3} \beta_{3}^{i}\right|\right) x_{2} \leq x_{i}
$$

Since $\left|\beta_{2}\right|>\left|\beta_{3}\right|$ and $\left|c_{3}\right|<1$ it follows that

$$
\lim _{i \rightarrow \infty} \frac{\left(c_{2} \beta_{2}^{i}-2\left|c_{3} \beta_{3}^{i}\right|\right) x_{2}}{\beta_{2}} \cdot \frac{2^{i+1}}{2^{i+1}}=c_{2} x_{2},
$$

which isn't 0 unless $x_{2}=0$. Since $x_{2}=0$ implies the trivial everywhere-zero measure we can assume that the limit exists and doesn't equal 0 , which by the limit comparison test shows that the series $\sum_{i=1}^{\infty} \frac{x_{i}}{2^{i+1}}$ diverges. This means that the density of the random map $T$ blows up in the $\epsilon$-neighborhoods of $\frac{1}{2}$ and 1 and $T$ has an infinite $\operatorname{acim} m=f \cdot \lambda$. The measure $m$ is supported on the whole interval $[0,1]$. The ergodicity of $m$ can be proved as in the Theorem 2.2.

Using Maple, we prepared a program which produced a histogram, shown in Fig. 3, of the 500000 iterations of random map $T$. It confirms that $T$-invariant density has singularities at $\frac{1}{2}$ and 1 . The width of the histogram boxes used was $1 / 1000$.

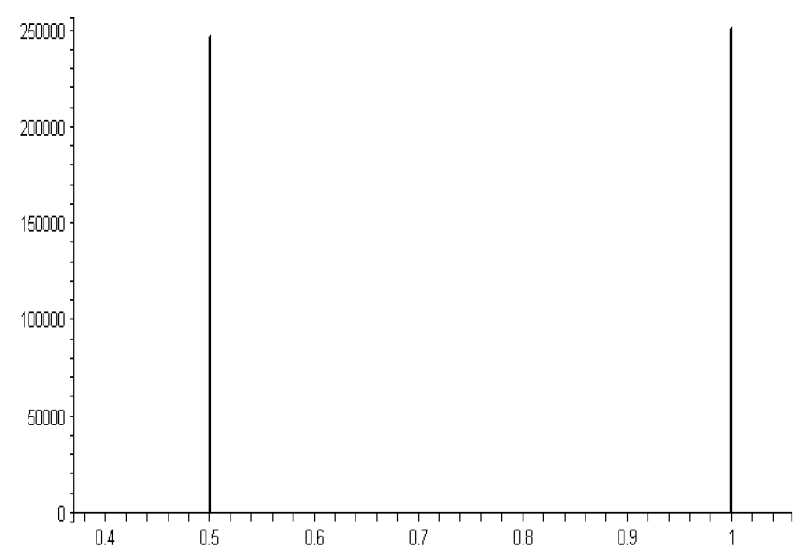

Figure 3: The histogram of 500000 points of a trajectory of random map $T$.

\section{REFERENCES}

[1] J. Aaronson, An Introduction to Infinite Ergodic Theory, Mathematical Surveys and Monographs, American Mathematical Society, 1997.

[2] A. Boyarsky, P. Góra, and M.S. Islam, Randomely chosen chaotic can give rice to nearly ordered behavior, Pysica D, 210 (2005). 
[3] G. Knight, S. Munday, Escape rates scaling in infinite measure preserving systems, J. Physics A: Math. Theor., 49, No. 085101 (2016).

[4] M. Kessebohmer, S. Munday, B.O. Stratmann, Strong renewal theorems and lyapunov spectra for a $\alpha$-Farey and $\alpha$-Luroth systems, Ergodic Theory and Dynamical Systems, 32, No. 5 (2012), 989-1017.

[5] J.M.R. Parrondo, G.P. Harmer, D. Abbott, New paradoxical games based on Brownian ratchets, Phys. Rev. Lett., 85 (2000), 5226-5229.

[6] A. Lasota, J.A. Yorke, On the existence of invariant measures for piecewise monotonic transformations, Trans. Amer. Math. Soc., 186 (1973), 481-488.

[7] S. Pelikan, Invariant densities for random maps of the interval, Proc. Amer. Math. Soc., 281 (1984), 813-825.

[8] P. Góra, Countably piecewise expanding transformations without absolutely continuous invariant measure, In: Proceedings of Stefan Banach Center - Semester on Dynamical Systems and Ergodic Theory, Warsaw 1986, PWN - Polish Scientific Publishers, 1989, 113-117.

[9] A. Boyarsky, P. Góra, Laws of Chaos, Birkhaüser, N. York, 1997.

[10] R. Mustafa, S. Kulenovic, O. Merino, Discrete Dynamical Systems and Difference Equations with Mathematica, CRC Press, C\&H, Boca Raton, 2002. 